\title{
Coordination of pricing and co-op advertising models in supply chain: A game theoretic approach
}

\author{
Amin Alirezaei* and Farid khoshAlhan
}

Department of Industrial Engineering, K. N. Toosi university of Technology, Tehran, Iran

\begin{tabular}{l}
\hline C H R O N I C L E \\
\hline Article history: \\
Received July 22013 \\
Received in revised format \\
September 72013 \\
Accepted September 152013 \\
Available online \\
September 212013 \\
\hline Keywords: \\
Cooperative advertising \\
Pricing \\
Supply chain \\
Duopolistic retailers \\
Game theory \\
Nash Equilibrium
\end{tabular}
A B S T R A C T

\begin{abstract}
Co-op advertising is an interactive relationship between manufacturer and retailer(s) supply chain and makes up the majority of marketing budget in many product lines for manufacturers and retailers. This paper considers pricing and co-op advertising decisions in two-stage supply chain and develops a monopolistic retailer and duopolistic retailer's model. In these models, the manufacturer and the retailers play the Nash, Manufacturer-Stackelberg and cooperative game to make optimal pricing and co-op advertising decisions. A bargaining model is utilized for determine the best pricing and co-op advertising scheme for achieving full coordination in the supply chain.
\end{abstract}

\section{Introduction}

Co-op advertising is, practically, an interactive relationship between a manufacturer and a retailer in which the manufacturer pays a portion of the retailer's local advertising costs; the fraction shared by the manufacturer is commonly referred to as the manufacturer's participation rate. Cooperative advertising is a coordination mechanism for advertising activities in a supply chain. In cooperative supply chain, the manufacturer may contributes part of advertising expenditures which are paid by retailers. Berger (1972) was the first to analyze co-op advertising issues between a manufacturer and a retailer mathematically. Berger's model was then extended by researchers in a variety of ways under different co-op advertising settings. The main reason for the manufacturer to use co-op advertising is to strengthen the image of the brand and to motivate immediate sales at the retail level. The manufacturer's national advertising is intended to influence potential consumers to consider its brand

* Corresponding author.

E-mail: aminalirezaei@yahoo.com (A. Alirezaei)

(C) 2014 Growing Science Ltd. All rights reserved. doi: $10.5267 / j . i j$ iec.2013.09.006 
and to help develop brand knowledge and preference. Retailer's local advertising is to stimulate consumer's buying behavior. Thus, Co-op advertising plays a significant role in the manufacturerretailer channel relationship. Brennan (1988) reports that in the personal computer industry; IBM offers a 50-50 split of advertising costs with retailers while Apple Computer pays $75 \%$ of the media costs.

Several studies on advertising efforts and pricing strategy have focused on distribution channels formed by one manufacturer and one retailer. Karray and Zaccour (2006) proposed a model to study the decision of a private label introduction for a retailer and its effects on the manufacturer. They showed that the private label introduction improves both the profit of the retailer, manufacturer and of the channel. Yue et al. (2006) studied the coordination of cooperative advertisement in a manufacturerretailer supply chain when the manufacturer offers price deductions to consumers. They showed that for any given price deduction, the total profit for the supply chain with cooperative scheme is always higher than without cooperation. He et al. (2009) modeled a one manufacturer- one retailer supply chain as a stochastic Stackelberg differential game; they consider the demand which depend on both retailer's price and advertising. Also Szmerekovsky and Zhang (2009) considered pricing and advertising dependent demand function in a two member supply chain and obtain ManufacturerStackelberg Equilibrium. Xie and Wei (2009) addressed channel coordination by seeking optimal cooperative advertising strategies and equilibrium pricing in a manufacturer-retailer distribution channel. They compared two models: a non-cooperative, leader-follower game and a cooperative game. They showed that cooperative model achieves better coordination by generating higher channel total profit than the non-cooperative one, lower retailer price to consumers, and the advertising efforts are higher for all channel members. They identified the feasible solutions to a bargaining problem where the channel members can determine how to divide the extra-profits generated by cooperation. Xie and neyret (2009) followed a similar approach; they compared the cooperative game optimal results and three of non-cooperative games including Nash game, Manufacturer-Stackelberg and RetailerStackelberg. SeyedEsfahani et al. (2011) applied these four games on the model of similar to one that proposed by (Xie, 2009) but relax the assumption of a linear price demand function by introducing a new parameter $v$ which can cause either a concave $(v>1)$ or linear $(v=1)$ or a convex $(v<1)$ curve. Aust and Buscher (2012) also considered one manufacturer-one retailer supply chain; they extend the model of SeyedEsfahani et al. (2011) and intended to relax assumption of equal margins by substitute the retail price $(p)$ into wholesales price and retailers margin $(p=m+w)$ to get better vision into the effect of market power on the distribution of channel profits.

Some other papers have been interested by a one manufacturer and two retailer's supply chain. Cachon and Lariviere (2005) studied revenue-sharing contracts in a general supply chain model with revenues determined by each retailer's purchase quantity and price. Yang and Zhou (2006) considered the pricing and quantity decisions of a two-echelon system with a manufacturer who supplies a single product to two competitive retailers. They analyzed the effects of the duopolistic retailer's different competitive behaviors (Cournot, Collusion and Stackelberg) on the optimal decisions of the manufacturer and the retailers. Wang et al. (2011) introduced one manufacturer-two retailer model in co-op advertising. They consider just advertising decision and suppose prices as constant parameters and adjust four possible non-cooperative games: Stackelberg-Cournot, in which the manufacturer and the duopolistic retailers play manufacturer-Stackelberg game, whereas the duopolistic retailers pursue Collusion behavior in the downstream market of the supply chain. Stackelberg-Collusion, in which the manufacturer and the duopolistic retailers play Vertical-Nash game and the duopolistic retailers obey Cournot behavior in the downstream market of the supply chain. Nash-Cournot, the manufacturer and the duopolistic retailers play Vertical-Nash game; the duopolistic retailers obey Cournot behavior in the downstream market of the supply chain. Nash-Collusion, in which the manufacturer and the duopolistic retailers play VerticalNash game; the duopolistic retailers pursue Collusion behavior in the downstream market of the supply chain. Jorgenson and Zaccour (2013) surveyed the literature on co-op advertising in marketing channels. The survey is divided into two main parts. The first one deals with co-op advertising in 
simple marketing channels having one manufacturer and one retailer only. The second one covers marketing channels more complex structure, having more than one player in each stage of supply chain.

Extant studies of cooperative advertising mainly consider a single-manufacturer-single-retailer channel structure. This can provide limited insights, because a manufacturer, in real practices, would frequently deal with multiple retailers at the same time. In order to examine the impact of the retailer's multiplicity on channel members' decisions and total channel efficiencies, this paper develops a monopolistic retailer and duopolistic retailer's model. In these models, the manufacturer and the retailers play the Nash, Manufacturer-Stackelberg and cooperative game to make optimal pricing and co-op advertising decisions. Our research is closely related to the one of Aust and Buscher (2012). We made some simplifications to their model by considering that there are no production costs for manufacturer and suppose that $(v=1)$. However, we enrich their model by considering two competing retailers and introduce a new demand function for each retailer's when local advertising of each retailer effect reversely on the other retailer demand. This extension enables us to study the case of competition between the retailers. In addition, we evaluate the impact of cooperation between all members of the supply chain on consumer's surplus and supply chains profit. Such comparisons are interesting and have not been done before by previous studies on supply chain.

The rest of the paper is organized as follows. Section 2 provides profit functions for both the manufacturer and monopolistic retailer based on the demand function with brand name investments and local advertising expenses. Section 3 obtains Nash and Stackelberg equilibrium when the manufacturer is the leader and the retailer is the follower. Pareto solution of channel obtains by solving cooperative game. Section 4 introduces the duopolistic retailer's model based on the new demand function. Section 5 introduces algorithms to gain Nash, Manufacturer-Stackelberg and cooperative equilibriums. Section 6 discusses the bargaining results to determine the shares of profits between the manufacturer and retailer. A simple contract is also provided to assure the profit sharing. Numerical example proposed in section 7. At the end, Managerial implications and Conclusion remarks are given in Section 8.

\section{Monopoly retailer}

In this section we define the assumption and notation to be used in the rest of paper and then introduce the monopoly retailer models. Consider a single-manufacturer-single-retailer channel in which the manufacturer sells certain product only through the retailer, and the retailer sells only the manufacturer's brand within the product class. Decision variables for the channel members are their advertising efforts, their prices (manufacturer's wholesale price and retailer's retail price) and the co-op advertising reimbursement policy. Denote by $(a)$ and $(A)$, respectively, the retailer's local advertising level and the manufacturer's national advertising investment. The consumer demand function depends on the retail price $(p)$ and the advertising levels $(a)$ and $(A)$ in a multiplicatively separable way like in Xie and Wei (2009) i.e.: $D(p, a, A)=g(p) \cdot h(a, A)$

Where $g(p)$ is linearly decreasing with respect to $(p)$ that is $g(p)=(\alpha-\beta p)$, and $h(a, A)$ is the function that Xie and Wei (2009) proposed to model advertising effects on sales in a static way. That is $h(a, A)=\left(k_{1} \sqrt{a}+k_{2} \sqrt{A}\right)$. Obviously, $h(a, A)$ is continuously differentiable, strictly increasing, and strictly (joint) concave with respect to $(a, A)$. According to Choi (1991), we introduce the retailer margin $(m)$ as a new decision variable with $(m=p-w)$ hence, we derive the following modified price and advertising dependent demand function in (1). By splitting the retail price $(p)$ into wholesale price $(w)$ and retailer margin $(m)$, the wholesale price also has an impact on the consumer demand.

$$
D(w, m, a, A)=(\alpha-\beta(m+w))\left(k_{1} \sqrt{a}+k_{2} \sqrt{A}\right)
$$


To implement co-op advertising, let Manufacturer shares portion $t \in[0,1]$ of Retailers local advertising $\operatorname{cost}(a)$. Denote by $(t)$ the fraction of the local advertising expenditure, which is the percentage the manufacturer agrees to share with the retailer. Under these assumptions, the profit of the manufacturer, the retailer and the system can be expressed as follows, respectively:

$$
\begin{aligned}
& \pi_{M}=w \cdot(\alpha-\beta(m+w))\left(k_{1} \sqrt{a}+k_{2} \sqrt{A}\right)-t \cdot a-A \\
& \pi_{R}=m \cdot(\alpha-\beta(m+w))\left(k_{1} \sqrt{a}+k_{2} \sqrt{A}\right)-(1-t) \cdot a \\
& \pi_{S}=\pi_{R}+\pi_{M}=p \cdot(\alpha-\beta p)\left(k_{1} \sqrt{a}+k_{2} \sqrt{A}\right)-a-A(\text { in which } p=m+w)
\end{aligned}
$$

In the next section, we analyzed the supply chain by game theoretic approach.

\section{Game theoretic analysis for monopoly model}

In the decentralized decision-making system, each entity of the supply chain maximizes its own profit without considering the profit of others. In the following, we will discuss how the manufacturer and the retailer determine separately their pricing and advertising policies under the three settings mentioned earlier, i.e.

\subsection{Nash game}

When the manufacturer and the retailer have the same decision power, they simultaneously and noncooperatively maximize their own profits. This situation is called a Nash game and the solution provided by this structure is called the Nash equilibrium. Definitely, the manufacturer's decision problem is:

$$
\begin{aligned}
& \operatorname{Max} \pi_{M}=w \cdot(\alpha-\beta(m+w)) \cdot\left(k_{1} \sqrt{a}+k_{2} \sqrt{A}\right)-t \cdot a-A \\
& \text { st }: 0 \leq t<1,0 \leq w<\frac{\alpha}{\beta}-m, 0 \leq A
\end{aligned}
$$

and the retailer's decision problem is:

$$
\begin{aligned}
& \text { Max } \pi_{R}=m \cdot(\alpha-\beta(m+w)) \cdot\left(k_{1} \sqrt{a}+k_{2} \sqrt{A}\right)-(1-t) \cdot a \\
& \text { st: } 0 \leq m<\frac{\alpha}{\beta}-w, 0 \leq a
\end{aligned}
$$

It is obvious that the optimal value of $t$ is zero because of its negative coefficient in the Manufacturer utility function. The first-order conditions for the manufacturer and the retailer are as following:

$$
\begin{aligned}
& \frac{\partial \pi_{M}}{\partial w}=(\alpha-\beta(m+w))\left(k_{1} \sqrt{a}+k_{2} \sqrt{A}\right)-\beta w\left(k_{1} \sqrt{a}+k_{2} \sqrt{A}\right), \frac{\partial \pi_{M}}{\partial A}=k_{2} w(\alpha-\beta(m+w)) / 2 \sqrt{A}-1 \\
& \frac{\partial \pi_{R}}{\partial m}=(\alpha-\beta(m+w))\left(k_{1} \sqrt{a}+k_{2} \sqrt{A}\right)-\beta m\left(k_{1} \sqrt{a}+k_{2} \sqrt{A}\right), \frac{\partial \pi_{R}}{\partial a}=k_{1} m(\alpha-\beta(m+w)) / 2 \sqrt{a}-(1-t)
\end{aligned}
$$

By noticing that $t$ should be zero under this situation and simultaneously solving Eq. (7) and Eq. (8); we can obtain the unique Nash equilibrium as shown in Eq. (9). (See Appendix1 for proof)

$$
m=\frac{\alpha}{3 \beta}, a=\frac{k_{1}^{2} \alpha^{4}}{324 \beta^{2}} w=\frac{\alpha}{3 \beta}, A=\frac{k_{2}^{2} \alpha^{4}}{324 \beta^{2}}, t=0
$$

\subsection{Manufacturer-Stackelberg game}

In a manufacturer and retailer supply chain, traditionally the manufacturer holds manipulative power, acts as the leader of the chain, and is followed by the retailers. In a leader-follower two-stage supply chain, the manufacturer usually anticipates the reactions of the retailer and decides its first move, and 
then prescribes the behavior of the retailer. In order to determine the Manufacturer-Stackelberg equilibrium, we first solve the retailer's decision problem (6) to find the best responses of $(m, a)$ to any given values Manufacturer's strategies; we can easily solved similar to Nash-game structure by solving Eq. (8), So the manufacturer's decision problem is:

$$
\begin{aligned}
& \operatorname{Max} \pi_{M}=w \cdot(\alpha-\beta(m+w)) \cdot\left(k_{1} \sqrt{a}+k_{2} \sqrt{A}\right)-t \cdot a-A \\
& \text { st : } m=\frac{(\alpha-\beta w)}{2 \beta}, a=\frac{k_{1}^{2}(\alpha-\beta w)^{3}}{64 \beta^{2}(1-t)^{2}}, 0 \leq t<1, w<\frac{\alpha}{\beta}-m, A \geq 0
\end{aligned}
$$

Since $\pi_{M}$ is a concave function of Manufacturer's decision variable (see Appendix A for proof), his reaction function can be derived from the first-order condition of Eq. (10)

$$
\begin{aligned}
& \frac{\partial \pi_{M}}{\partial A}=k_{2} w\left(\alpha-\beta\left(\frac{(\alpha-\beta w)}{2 \beta}+w\right)\right) / 2 \sqrt{A}-1 \\
& \frac{\partial \pi_{M}}{\partial t}=\frac{k_{1}^{2} t(\alpha-\beta w)^{4}}{32 \beta^{2}(1-t)^{3}}-\frac{k_{1}^{2}(\alpha-\beta w)^{4}}{64 \beta^{2}(1-t)^{2}}-\frac{k_{1}^{2} w(\alpha-\beta w)^{3}}{16 \beta(1-t)^{2}} \\
& \frac{\partial \pi_{M}}{\partial w}=\left(k_{2} \sqrt{A}+\frac{k_{1}^{2}(\alpha-\beta w)^{2}}{8 \beta(1-t)}\right)\left(\frac{\alpha}{2}-\beta w\right)-\frac{k_{1}^{2} t(\alpha-\beta w)^{3}}{16 \beta(1-t)^{2}}-\frac{k_{1}^{2} w(\alpha-\beta w)^{2}}{8(1-t)}
\end{aligned}
$$

We can easily solve the Eqs. (11-12) and find $A, t$ according to $w$ :

$$
m=\frac{(\alpha-\beta w)}{2 \beta}, a=\frac{k_{1}^{2}(3 \beta w+\alpha)^{2}(\alpha-\beta w)^{2}}{256 \beta^{2}} A=\frac{k_{2}^{2} w^{2}(\alpha-\beta w)^{2}}{16}, t=\frac{5 \beta w-\alpha}{3 \beta w+\alpha}
$$

We failed to analytically solve the Eq. (13) for the manufacturer's wholesale price in the Stackelberg manufacturer case. In order to solve Eq. (13) numerically, we substitute the variable $(m, a, A, t)$ from Eq. (14) into Eq. (13). To obtain the manufacturer's price $w$, for each group of examples we use MATLAB to solve these equations and obtain the Manufacturer-Stackelberg equilibrium, to check the upper and lower bound we use the simple algorithm, which shown in rest. (See Appendix2 for proof)

Step 1 Find the solution of Eq. (13) and check it in its bounds, if it's true placed in $w^{*}$ else placed upper bound in $w^{*}$

Step 2: $\quad$ Based on $w^{*}$ find the solution of $A, t$ for Manufacturer from Eq. (14)

Step 3: Based on Manufacturer's decisions find the solution of Retailer from Eq. (14)

\subsection{Cooperative game}

Here we try to reach the optimal profit of the supply chain $\left(\pi_{s}\right)$ by defining the members' strategies. The channel's profit is described by $\pi_{S}=\pi_{R}+\pi_{M}$ is that shown on problem (15) and depends only on $p$, $a$ and $A$. We hence have the following optimization problem:

$\operatorname{Max} \pi_{S}=p \cdot(\alpha-\beta \cdot p) \cdot\left(k_{1} \sqrt{a}+k_{2} \sqrt{A}\right)-a-A$

st : $0 \leq p<\frac{\alpha}{\beta}, 0<a, A$

This equation can easily be solved by taking the three first order equations equal to zeros. Specifically, we have: 
$\frac{\partial \pi_{S}}{\partial p}=(\alpha-\beta p)\left(k_{1} \sqrt{a}+k_{2} \sqrt{A}\right)-\beta p\left(k_{1} \sqrt{a}+k_{2} \sqrt{A}\right), \frac{\partial \pi_{s}}{\partial a}=\frac{k_{1} p(\alpha-\beta p)}{2 \sqrt{a}}-1, \frac{\partial \pi_{S}}{\partial A}=\frac{k_{2} p(\alpha-\beta p)}{2 \sqrt{A}}-1$

For solving the model, we should calculate extremum nodes. Regard to the strictly concavity of objective function, extremum node will be the optimal one if it satisfies constraints; else, we should check boundary nodes to find the optimal solution. In the first model, this node (boundary node or extremum node) is satisfying constraints and because of the hessian matrix it is an optimal solution. These equations lead to the solution which shown in Eq. (17)

$$
p=\frac{\alpha}{2 \beta}, a=\frac{k_{1}^{2}}{64} \cdot \frac{\alpha^{4}}{\beta^{2}}, A=\frac{k_{2}^{2}}{64} \cdot \frac{\alpha^{4}}{\beta^{2}}
$$

As can be seen the solution of optimal retail price is located within the bound. In the next section, we formalize our duopolistic retailer's model which allows for varying profit margins. (See Appendix 3 for proof).

\section{Duopolistic retailers model}

In this section we model the relationship between monopolistic manufacturer and duopolistic retailers, this model for first time will consider cooperative advertising issues of a two echelon supply chain in which a monopolistic manufacturer sells its product through duopolistic retailers. The manufacturer invests in the product's national brand name advertising in order to take potential customers from the awareness of the product to the purchase consideration. On the other hand, the manufacturer would like retailers to invest in local advertising in the hope of driving potential customers further to the stage of desire and action. Before establishing the models, we give notations used in this model in Table 1.

Table 1

Notation for monopolistic-manufacturer duopolistic-retailers model

\begin{tabular}{ll}
\hline$D_{i}(p, a, A)$ & Demand function \\
$\alpha_{i}$ & Potential demand of retailer $i$ \\
$\beta$ & Price sensitivity \\
$\gamma$ & Competitors prices \\
$k_{1}$ & Effectiveness of local advertising \\
$k_{2}$ & Effectiveness of global advertising \\
$k_{3}$ & Effectiveness of compete retailer's local advertising \\
$p_{i}$ & Retail price \\
$m_{i}$ & (Retailer $i$ Decision variable) Retailer profit margin \\
$a_{i}$ & (Retailer $i$ Decision variable) Local advertising expenditure \\
$w$ & (Manufacturer Decision variable) wholesale price \\
$A$ & (Manufacturer Decision variable) Global advertising expenditure \\
$t_{i}$ & (Manufacturer Decision variable) Advertising participation rate $\left(0 \leq t_{i}<1\right)$ \\
$\pi_{M}$ & Manufacturer's profit function \\
$\pi_{R_{i}}$ & Retailer's profit function \\
$\pi_{S}$ & Supply chain's profit function \\
\hline
\end{tabular}

We consider one manufacturer-two retailers distribution channel in which both retailers sell only the manufacturers brand within the product class. Assume that different retailers are geographically related, so there is intra-brand competition between two retailers. This assumption captures the real situation when a manufacturer's marketing channels are competitive between two retailers. Decision variables for the manufacturer are the national advertising expenditure $(A)$, the participation rate for 
each retailer $\left(t_{i} i=1,2\right)$ and the whole sale price to retailers $w$. The decision variables for the retailers are their margin profits $\left(m_{i} \quad i=1,2\right)$; and the local advertising expenditures $\left(a_{i} \quad i=1,2\right)$.

The reason why the above functions are adopted to depict the retailers' demand is twofold. On one hand, this type of demand form has been successively used in one manufacturer-one-retailer channel by Xie and Wei (2009), Aust and Buscher (2012). On the other hand, the theory of industrial organization has pointed out that under the case with two competitive retailers, one party's advertising effort will decrease the other's share of the marketing demand (see Luo (2006)). We assume the resulting consumer demand for retailer $R_{i}, D_{i}=D_{i}\left(p_{i}, a, A\right) \quad i=1,2$ often called the sales response function, is jointly determined by both the prices and advertises. There is a substantial literature on the estimation of the sales response function with respect to pricing and co-op advertising investments. We extend the model of section 2 by considering negative effectiveness of price and advertisement of competitor retailer. The manufacturer uses brand advertising to increase consumer's interest and demand for the product. Consumer's demand $D_{i}$ for the product proposed by retailer $i$ depend on the retail prices and the advertising level as:

$$
D_{i}\left(p_{i}, p_{-i}, a_{i}, a_{-i}, A\right)=g_{i}\left(p_{i}, p_{-i}\right) \cdot h\left(a_{i}, a_{-i}, A\right),
$$

where $g_{i}\left(p_{i}, p_{-i}\right)$ and $h\left(a_{i}, a_{-i}, A\right)$ reflect the impact of the retail prices and the brand advertising expenditures on the demand of retailer $i$; By splitting the retail price $\left(p_{i}=w+m_{i} i=1,2\right)$ into wholesales price $(w)$ and retailer $R_{i} \operatorname{margin}\left(m_{i}\right)$, as also shown on section 2 , we generate a demand function as below:

$$
\begin{aligned}
& g_{i}\left(m_{i}, m_{-i}, w\right)=\left(\alpha_{i}-\beta \cdot\left(w+m_{i}\right)+\gamma \cdot\left(w+m_{-i}\right)\right) \\
& h\left(a_{i}, a_{-i}, A\right)=\left(k_{1} \sqrt{a_{i}}+k_{2} \sqrt{A}-k_{3} \sqrt{a_{-i}}\right)
\end{aligned}
$$

So the demand function for each retailer is:

$$
D_{i}\left(m_{i}, m_{-i}, w, a_{i}, a_{-i}, A\right)=\left(\alpha_{i}-\beta\left(w+m_{i}\right)+\gamma\left(w+m_{-i}\right)\right) \cdot\left(k_{1} \sqrt{a_{i}}+k_{2} \sqrt{A}-k_{3} \sqrt{a_{-i}}\right)
$$

From notations and assumptions above, we can easily calculate the profit functions for one manufacturer, two retailers and the supply chain system respectively as follows.

$$
\begin{aligned}
& \pi_{m}=w \cdot \sum_{i=1,2}\left(\alpha_{i}-\beta \cdot\left(w+m_{i}\right)+\gamma \cdot\left(w+m_{-i}\right)\right) \cdot\left(k_{1} \sqrt{a_{i}}+k_{2} \sqrt{A}-k_{3} \sqrt{a_{-i}}\right)-\sum_{i=1,2} t_{i} \cdot a_{i}-A \\
& \pi_{r_{i}}=m_{i} \cdot\left(\alpha_{i}-\beta \cdot\left(w+m_{i}\right)+\gamma \cdot\left(w+m_{-i}\right)\right) \cdot\left(k_{1} \sqrt{a_{i}}+k_{2} \sqrt{A}-k_{3} \sqrt{a_{-i}}\right)-\left(1-t_{i}\right) \cdot a_{i} \\
& \pi_{s}=\pi_{m}+\sum_{i=1,2} \pi_{r_{i}}=\sum_{i=1,2} p_{i} \cdot\left(\alpha_{i}-\beta \cdot p_{i}+\gamma \cdot p_{-i}\right) \cdot\left(k_{1} \sqrt{a_{i}}+k_{2} \sqrt{A}-k_{3} \sqrt{a_{-i}}\right)-\sum_{i=1,2} a_{i}-A
\end{aligned}
$$

\section{Game theoretic analysis for duopoly model}

In this section, similar to section 3, three game-theoretic models based on two non-cooperative games including Nash and Stackelberg-manufacturer with one cooperative is discussed. Because of models difficulty parametric solution could not obtain, so we introduce algorithms to each game structure.

\subsection{Nash game}

To determine the Nash Equilibrium, manufacturer and retailer's decision problems are solved separately. We apply a similar approach as proposed in section 3 but unfortunately we can't solve this model parametrically, so we introduced a repetitive algorithm that applied for two models. For the monopolistic model; the solution obtain from new algorithm is similar to parametric solution obtained in section 3.1. So we can employ this algorithm for duopolistic retailer model. It is obvious that the 
optimal value of $t_{i}$ is zero because of its negative coefficient in the Manufacturer utility function. The first-order conditions for the manufacturer and the retailer are as following:

$$
\begin{aligned}
& \frac{\partial \pi_{M}}{\partial w}=\sum_{i=1.2}\left(\alpha_{i}-\beta m_{i}+m_{-i}+2(\gamma-\beta) w\right) \cdot\left(k_{1} \sqrt{a_{i}}+k_{2} \sqrt{A}-k_{3} \sqrt{a_{-i}}\right) \\
& \frac{\partial \pi_{M}}{\partial A}=w \cdot k_{2} \sum_{i=1,2} \frac{\left(\alpha_{i}-\beta \cdot\left(w+m_{i}\right)+\gamma \cdot\left(w+m_{-i}\right)\right)}{2 \sqrt{A}}-1 \\
& \frac{\partial \pi_{R_{i}}}{\partial m_{i}}=\left(\alpha_{i}-\beta \cdot\left(w+m_{i}\right)+\gamma \cdot\left(w+m_{-i}\right)-\beta m_{i}\right) \cdot\left(k_{1} \sqrt{a_{i}}+k_{2} \sqrt{A}-k_{3} \sqrt{a_{-i}}\right) \quad i=1,2 \\
& \frac{\partial \pi_{R_{i}}}{\partial a_{i}}=\frac{k_{1} m_{i}\left(\alpha_{i}-\beta \cdot\left(w+m_{i}\right)+\gamma \cdot\left(w+m_{-i}\right)\right)}{2 \sqrt{a_{i}}}-\left(1-t_{i}\right) \quad i=1,2
\end{aligned}
$$

Under this situation and simultaneously solving Eqs. (25-28); we can obtain the unique Nash equilibrium as shown in Eq. (29) and Eq. (30).

$$
\begin{aligned}
& \left.w=\frac{\sum_{i=1,2}\left(\alpha_{i}-\beta m_{i}+\gamma m_{-i}\right) \cdot\left(k_{1} \sqrt{a_{i}}+k_{2} \sqrt{A}-k_{3} \sqrt{a_{-i}}\right)}{\sum_{i=1,2} 2(\beta-\gamma)\left(k_{1} \sqrt{a_{i}}+k_{2} \sqrt{A}-k_{3} \sqrt{a_{-i}}\right.}\right), A=\left(\frac{k_{2} w}{2} \sum_{i=1,2}\left(\alpha_{i}-\beta \cdot\left(w+m_{i}\right)+\gamma \cdot\left(w+m_{-i}\right)\right)\right)^{2}, t_{i}=0 i=1,2 \\
& m_{i}=\frac{\left(\alpha_{i}+\gamma m_{-i}+(\gamma-\beta) \cdot w\right)}{2 \beta}, a_{i}=\frac{k_{1}^{2} m_{i}^{2}}{4}\left(\alpha_{i}-\beta \cdot\left(w+m_{i}\right)+\gamma \cdot\left(w+m_{-i}\right)\right)^{2} \quad i=1,2
\end{aligned}
$$

We give the following solution algorithm to compute the equilibrium of the Nash game $X$ is denoted as the strategy set of the supply chain member Thus $X_{M}$ and $X_{R_{i}}$ are the strategy profile sets of the manufacturer and retailer $i$ strategies; respectively. We introduce the quadratic measure for the completion of algorithm, if $\Delta \pi_{S}=\left(\pi_{S}^{*}-\pi_{S}^{0}\right)^{2}$ is lower than $\varepsilon$ algorithm is accomplished and available solution is close enough to equations solution. We present the following repetitive algorithm for solving the non-cooperative game model:

Step 0 Give the initial strategy profile for the manufacturer and retailers $X^{0}=\left(m^{0}, a^{0}, w^{0}, A^{0}\right)$ in the strategy profile set $X$.

Step 1: For the manufacturer based on $\left(X_{R_{i}}^{0} i=1,2\right)$ the optimal reaction is $X_{M}^{*}=\left(w^{*}, A^{*}\right)$ in the strategy profile set $X^{*}$.

Step 2: For the retailer1 based on $\left(X_{2}^{0}\right.$ and $\left.X_{M}^{*}\right)$ the optimal reaction is $X_{R_{1}}^{*}=\left(m_{1}^{*}, a_{1}^{*}\right)$ in the strategy profile set $X^{*}$.

Step 3: For the retailer2 based on $\left(X_{1}^{*}\right.$ and $\left.X_{M}^{*}\right)$ the optimal reaction is $X_{R_{2}}^{*}=\left(m_{2}^{*}, a_{2}^{*}\right)$ in the strategy profile set $X^{*}$.

Step 4: For the whole supply chain, find out $\pi_{S}^{*}$ and $\pi_{S}^{*}$ based on $X^{*}$ and $X^{0}$; respectively. If $\Delta \pi_{S}=\left(\pi_{S}^{*}-\pi_{S}^{0}\right)^{2}<\varepsilon$ Nash equilibrium is obtain, Output the optimal results and stop. Else $X^{0}=X^{*}$ and go to step 1 . ( $\varepsilon$ is very small positive number)

\subsection{Manufacturer-Stackelberg game}

Now we confer more power to the manufacturer in order to analyze tradition supply chain where the manufacturer has manipulative power. Similar to section 3.2 we use Stackelberg equilibrium to solve 
this situation. Officially, we first solve the decision problem of the retailers to identify their response function; retailer's decision problem is identical to retailer's problem in previous section, as well as their response function:

$$
\begin{aligned}
& m_{i}=\frac{\left(\alpha_{i}+m_{-i}+(\gamma-\beta) \cdot w\right)}{2 \beta} \quad i=1,2 \\
& a_{i}=\frac{k_{1}^{2} m_{i}^{2}}{4\left(1-t_{i}\right)^{2}}\left(\alpha_{i}-\beta \cdot\left(w+m_{i}\right)+\gamma \cdot\left(w+m_{-i}\right)\right)^{2} \quad i=1,2
\end{aligned}
$$

After solving Eqs. (31) and substituting them into Eq. (32) and then substituting $m_{i}, a_{i} \quad i=1,2$ into $\pi_{m}$ we can formulate the manufacturer decision problem:

$$
\begin{aligned}
& \text { Max } \pi_{m}=w \cdot \sum_{i=1,2}\left(\alpha_{i}-\beta \cdot\left(w+m_{i}\right)+\gamma \cdot\left(w+m_{-i}\right)\right) \cdot\left(k_{1} \sqrt{a_{i}}+k_{2} \sqrt{A}-k_{3} \sqrt{a_{-i}}\right)-\sum_{i=1,2} t_{i} \cdot a_{i}-A \\
& s t: a_{i}=\frac{k_{1}^{2}}{4\left(1-t_{i}\right)^{2}}\left(\frac{2 \alpha_{i} \beta+\alpha_{-i} \gamma+w\left(\gamma^{2}+\beta \gamma-2 \beta^{2}\right)}{4 \beta^{2}-\gamma^{2}}\right)^{2}\left(\frac{2 \beta^{2} \alpha_{i}+\alpha_{-i} \beta \gamma+2 \beta^{2} \gamma-2 \beta^{3}}{4 \beta^{2}-\gamma^{2}}\right)^{2} \quad i=1,2 \\
& m_{i}=\frac{2 \alpha_{i} \beta+\alpha_{-i} \gamma+w\left(\gamma^{2}+\beta \gamma-2 \beta^{2}\right)}{4 \beta^{2}-\gamma^{2}} \quad i=1,2 \quad 0 \leq t_{i}<1 i=1,2 \quad 0 \leq w<\frac{\alpha}{\beta}-\operatorname{Max}_{i}\left\{m_{i}\right\} A \geq 0
\end{aligned}
$$

The game is a leader-follower one: the manufacturer chooses his decision variables, and then the retailers choose their retail prices. This game is solved backward to get a sub game-perfect Nash equilibrium. Since $\pi_{M}$ is a concave function of Manufacturer's decision variable, his reaction function can be derived from the first-order condition of Eq. (33).

$$
\frac{\partial \pi_{M}}{\partial w}=0, \frac{\partial \pi_{M}}{\partial A}=0, \frac{\partial \pi_{M}}{\partial t_{i}}=0 \quad i=1,2
$$

Similar to section 3.2 we failed to analytically solve the Eq. (34) for the manufacturer's wholesale price in the Stackelberg manufacturer case. In order to solve Eqs. (34) numerically, we substitute the variable $\left(m_{i}, a, A, t_{i}\right)$. To obtain the manufacturer's price $w$, and hen with substituting it into $\left(m_{i}, a, A, t_{i}\right)$ for each group of examples we use MATLAB to solve these equations and obtain the Manufacturer-Stackelberg equilibrium to check the upper and lower bound we use the simple algorithm, which shown in rest. (See Appendix2 for proof)

\footnotetext{
Step 1 Find the solution of $\frac{\partial \pi_{M}}{\partial w}=0$ and check it in its bounds, if it's true placed in $w^{*}$ else placed upper bound in $w^{*}$
}

Step 2: Based on $w^{*}$ find the solution of $A, t_{i}$ for Manufacturer from $\frac{\partial \pi_{M}}{\partial A}=0, \frac{\partial \pi_{M}}{\partial t_{i}}=0$

Step 3: Based on Manufacturer's decisions find the solution of Retailers from $(31,32)$

\subsection{Cooperative game}

Consider now a situation where both the manufacturer and the duopolistic retailers are prepared to cooperate to pursue the optimal pricing and advertising policies. Therefore, unlike in the decentralized case, the objective in this setting is to maximize the total profit of the system. That is: 


$$
\begin{aligned}
& \operatorname{Max} \pi_{s}=\sum_{i=1,2} p_{i} \cdot\left(\alpha_{i}-\beta \cdot p_{i}+\gamma \cdot p_{-i}\right) \cdot\left(k_{1} \sqrt{a_{i}}+k_{2} \sqrt{A}-k_{3} \sqrt{a_{-i}}\right)-\sum_{i=1,2} a_{i}-A \\
& \text { st } 0 \leq p_{i}<\frac{\alpha_{i}}{\beta} \quad i=1,2 \quad 0 \leq a_{i} \quad i=1,2 \quad 0 \leq A
\end{aligned}
$$

By solving the first order condition of $\pi_{s}$ with respect to $p_{i}, a_{i}, A$ one has:

$$
\begin{aligned}
\frac{\partial \pi_{s}}{\partial p_{i}} & =\left(\alpha_{i}-2 \beta p_{i}+p_{-i}\right)\left(k_{1} \sqrt{a_{i}}+k_{2} \sqrt{A}-k_{3} \sqrt{a_{-i}}\right)+p_{-i}\left(k_{1} \sqrt{a_{-i}}+k_{2} \sqrt{A}-k_{3} \sqrt{a_{i}}\right) \quad i=1,2 \\
\frac{\partial \pi_{s}}{\partial a_{i}} & =\frac{k_{1} p_{i}\left(\alpha_{i}-\beta p_{i}+\not p_{-i}\right)-k_{3} p_{-i}\left(\alpha_{-i}-\beta p_{-i}+\gamma p_{i}\right)}{2 \sqrt{a_{i}}}-1 \quad i=1,2 \\
\frac{\partial \pi_{s}}{\partial A} & =\sum_{i=1,2} \frac{k_{2} p_{i}\left(\alpha_{i}-\beta p_{i}+\gamma p_{-i}\right)}{2 \sqrt{A}}-1
\end{aligned}
$$

In this model, because of the problem's structure and this model's similarity to the first one, it can be predictable that, the extremum node will satisfy constraints. For assuring this, we checked several instances and in all of these instances this node satisfies all constraints.

From (Eqs. (36-38)) one can easily drive:

$$
\begin{aligned}
& p_{i}=\frac{\left(\alpha_{i}+\gamma p_{-i}\right)\left(k_{1} \sqrt{a_{i}}+k_{2} \sqrt{A}-k_{3} \sqrt{a_{-i}}\right)+p_{-i}\left(k_{1} \sqrt{a_{-i}}+k_{2} \sqrt{A}-k_{3} \sqrt{a_{i}}\right)}{2 \beta\left(k_{1} \sqrt{a_{i}}+k_{2} \sqrt{A}-k_{3} \sqrt{a_{-i}}\right)} \quad i=1,2 \\
& a_{i}=\frac{1}{4}\left(k_{1} p_{i}\left(\alpha_{i}-\beta p_{i}+\gamma p_{-i}\right)-k_{3} p_{-i}\left(\alpha_{-i}-\beta p_{-i}+\gamma p_{i}\right)\right)^{2} \quad i=1,2 \\
& A=\left(\sum_{i=1,2} \frac{k_{2} p_{i}\left(\alpha_{i}-\beta p_{i}+\gamma p_{-i}\right)}{2}\right)^{2}
\end{aligned}
$$

But we cannot solve these equations parametric, so we use the algorithm who describe in section 5.1 to obtain optimal solution of the whole channel, and obtain decision variables value, and profit of supply chain. In next section, we determined a bargaining model to share extra-profit between the supply chain members.

\section{A bargaining model}

Bargaining models are usually used in literature to find a suitable division of funds between two or more players. The results depend both on the underlying utility functions of the players and on the selected bargaining model. For instance Xie and Wei (2009), SeyedEsfahani et al. (2011) used power function of type $u(x)=x^{\lambda}$ to determine the player's convenience in combination with the Nash bargaining model Nash (1950). We assume that all players are rational, self-interested and risk natural.

In this paper, we will use bargaining model which similar to that Aust and Buscher (2012) presented. The extra-profits accrued from the cooperative game relative to the non-cooperative games can be expressed as $\Delta \pi_{S}=\pi_{S}^{*}-\left(\sum_{i=1,2} \pi_{R_{i}}^{\max }+\pi_{M}^{\max }\right)$, with $\pi_{S}^{*}$ being the channel profits under the cooperative game; $\pi_{M}^{\max }$ and $\pi_{R_{i}}^{\max }$ respectively being the maximum profits of manufacturer and retailer $i$ under the noncooperative situations. The extra-profits $\Delta \pi_{S}$ are greater than zero. Now we discuss how such extraprofits should be jointly shared between the manufacturer and the retailer(s). In order to ensure that all players are willing to participate in a cooperative rather than a non-cooperative relationship, we face a bargaining problem over $0<w \leq \operatorname{Min}_{i}\left\{p_{i}\right\} \quad i=1,2$ and $\quad 0 \leq t_{i}<1 \quad i=1,2$ subject to $\Delta \pi_{M}=\pi_{M}^{*}-\pi_{M}^{\max }$ and $\Delta \pi_{R_{i}}=\pi_{R_{i}}^{*}-\pi_{R_{i}}^{\max } \quad i=1,2$ where $\pi_{M}^{*}$ and $\pi_{R_{i}}^{*}$ are manufacturer and retailer $i$ 's profit, respectively, under 
the cooperative game. That is $\Delta \pi_{M}$ and $\Delta \pi_{R_{i}}$ are extra-profits that can be made by manufacturer and retailer $i$, respectively and obviously $\Delta \pi_{S}=\Delta \pi_{M}+\Delta \pi_{R_{i}}$. We can formulate the bargaining model by $U_{s}=U_{M}\left(\Delta \pi_{M}\right)^{\mu_{M}} \prod_{i=1,2} U_{R_{i}}\left(\Delta \pi_{R_{i}}\right)^{\mu_{R_{i}}}$ Where $\mu_{M}$ and $\mu_{R_{i}}$ are positive parameter with $\left(\mu_{M}+\sum_{i=1,2} \mu_{R_{i}}=1\right)$ which reflect each players bargaining power. And where $\lambda_{M}$ and $\lambda_{R_{i}}$ are positive parameters reflecting the players risk attitude, we derive the following optimization problem:

$$
\begin{aligned}
& \operatorname{Max} U_{s}=\Delta \pi_{M}^{\lambda_{M} \mu_{M}} \prod_{i=1,2} \Delta \pi_{R_{i}}^{\lambda_{R_{i}} \mu_{R_{i}}} \\
& s t: \Delta \pi_{S}=\Delta \pi_{M}+\sum_{i=1,2} \Delta \pi_{R_{i}} \quad \Delta \pi_{M}, \Delta \pi_{R_{i}}>0 \quad i=1,2
\end{aligned}
$$

Nash bargaining model leads to the following division of profits:

$$
\Delta \pi_{M}=\frac{\lambda_{M} \mu_{M}}{\lambda_{M} \mu_{M}+\sum_{i=1,2} \lambda_{R_{i}} \mu_{R_{i}}} \cdot \Delta \pi_{S} \text { and } \Delta \pi_{R_{i}}=\frac{\lambda_{R_{i}} \mu_{R_{i}}}{\lambda_{M} \mu_{M}+\sum_{i=1,2} \lambda_{R_{i}} \mu_{R_{i}}} . \Delta \pi_{S} \quad i=1,2
$$

When $\Delta \pi_{M}$ and $\Delta \pi_{R_{i}} \quad i=1,2$ have been determined, the manufacturer and retailers can position themselves to make decision about $w$ and $t_{i} \quad i=1,2$ to obtain the profits equal to $\pi_{M}^{*}$ and $\pi_{R_{i}}^{*} i=1,2$ respectively. For each $w$ that manufacturer sales products he can determine participation rate for monopolistic and duopolistic retailer(s) from Eq. (44) and Eq. (45), respectively.

$t=B+C w$ whereas: $B=\frac{\pi_{R}-p(\alpha-\beta \cdot p) \cdot\left(k_{1} \sqrt{a}+k_{2} \sqrt{A}\right)+a}{a}, C=\frac{(\alpha-\beta \cdot p) \cdot\left(k_{1} \sqrt{a}+k_{2} \sqrt{A}\right)}{a}$

$t_{i}=B_{i}+C_{i} w \quad i=1,2$ whereas:

$B_{i}=\frac{\pi_{R_{i}}-p_{i}\left(\alpha_{i}-\beta\left(p_{i}\right)+\gamma\left(p_{-i}\right)\right) \cdot\left(k_{1} \sqrt{a_{i}}+k_{2} \sqrt{A}-k_{3} \sqrt{a_{-i}}\right)+a_{i}}{a_{i}}, C_{i}=\frac{\left(\alpha_{i}-\beta\left(p_{i}\right)+\gamma\left(p_{-i}\right)\right) \cdot\left(k_{1} \sqrt{a_{i}}+k_{2} \sqrt{A}-k_{3} \sqrt{a_{-i}}\right)}{a_{i}}$

If we assume that $\left(\mu_{M}=\mu_{R_{1}}=\mu_{R_{2}}=1 / 3\right)$ and one player is more risk-seeking than other players, i.e. $\left(\lambda_{M}>\lambda_{R_{i}} \quad i=1,2\right)$ he will receive the bigger fraction of the extra-profit. Now we set $\lambda_{M}=\lambda_{R_{1}}=\lambda_{R_{2}}=$ const . In order to analyze the effect of the bargaining power parameters $\mu_{M}$ and $\mu_{R_{i}}$. As expected, an equal bargaining power of all players results in a homogeneous division and otherwise the player with the higher bargaining power will be able to get bigger fraction of profit.

\section{Numerical example}

To demonstrate the application of the proposed game models and solution algorithms we will examine it through numerical experiments. The experiments are implemented in the following manner. First, for all parameter of the models, we extract randomly a value out of its given interval, which is shown in Table 2. We extract randomly more than 100 groups of values of the parameters in total in the experiment. Then we calculate the equilibrium solution of two models in the tree settings based on this group of extracted values of all parameters. Our remarks below are obtained based on the computational results of all groups. For shortness, we pick arbitrarily six from all groups, in which the values of parameters are listed in Table 3 and table 5 for monopolistic retailer and duopolistic retailers' model; respectively to illustrate our observations intuitively. 
Table 4 and Table 6 show Nash equilibrium, Manufacturer-Stackelberg Equilibrium and cooperative equilibrium solutions.

Table 2

The ranges of parameters

\begin{tabular}{lllllll}
\hline Parameters & $\alpha_{1}, \alpha_{2}$ & $\beta$ & $\gamma$ & $k_{1}$ & $k_{2}$ & $k_{3}$ \\
\hline Ranges & {$[100000-130000]$} & {$[30-45]$} & {$[5-20]$} & {$[0.0004-0.0005]$} & {$[0.0003-0.0005]$} & {$[0.0001-0.0002]$} \\
\hline
\end{tabular}

\section{1 monopolistic retailer model}

For the monopolistic retailer model we use these six groups as shown in Table 3 and obtain the solution as reported in Table 4.

Table 3

Monopoly model parameters

\begin{tabular}{lllllll}
\hline & Example 1 & Example 2 & Example 3 & Example 4 & Example 5 & Example 6 \\
\hline$\alpha$ & 110486 & 231202 & 218043 & 117686 & 224880 & 110696 \\
$\beta$ & 41.9926 & 40.8178 & 30.0341 & 40.254 & 35.2557 & 42.6021 \\
$k_{1}$ & 0.0004977 & 0.0004871 & 0.000443 & 0.0004873 & 0.0004194 & 0.0004557 \\
$k_{2}$ & 0.0003016 & 0.0003466 & 0.0003252 & 0.0004656 & 0.0003358 & 0.0004993 \\
\hline
\end{tabular}

\section{Table 4}

Decisions and profits of players in monopolistic retailer model

\begin{tabular}{|c|c|c|c|c|c|c|c|}
\hline & & Example 1 & Example 2 & Example 3 & Example 4 & Example 5 & Example 6 \\
\hline \multirow{7}{*}{$\begin{array}{l}\overline{\bar{w}} \\
\overline{\tilde{z}}\end{array}$} & $w$ & 877 & 1888 & 2420 & 975 & 2126 & 866 \\
\hline & $A$ & 23724722 & 635886004 & 817890971 & 79206725 & 716079136 & 63656656 \\
\hline & $\pi_{M}$ & 152936987 & 3147705369 & 3853401810 & 252730478 & 2950096190 & 169706184 \\
\hline & $m$ & 877 & 1888 & 2420 & 975 & 2126 & 866 \\
\hline & $a$ & 64606133 & 1255909682 & 1517755420 & 86761876 & 1117008527 & 53024764 \\
\hline & $\pi_{R}$ & 112055577 & 2527681690 & 3153537361 & 245175326 & 2549166798 & 180338075 \\
\hline & $\pi_{S}$ & 264992565 & 5675387059 & 7006939171 & 497905804 & 5499262988 & 350044259 \\
\hline \multirow{8}{*}{ 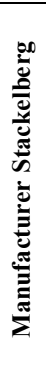 } & $w$ & 990 & 2196 & 2832 & 1205 & 2533 & 1102 \\
\hline & $A$ & 26457216 & 725631528 & 937547831 & 94152965 & 831133771 & 76892234 \\
\hline & $t$ & 0.4139 & 0.4339 & 0.4380 & 0.4743 & 0.4497 & 0.4931 \\
\hline & $\pi_{M}$ & 170622119 & 3513981741 & 4303148454 & 283947539 & 3298636365 & 191818873 \\
\hline & $m$ & 821 & 1734 & 2214 & 859 & 1923 & 748 \\
\hline & $a$ & 144164903 & 2788350212 & 3365600623 & 189794575 & 2467502594 & 114926639 \\
\hline & $\pi_{R}$ & 128372208 & 2724670635 & 3357357397 & 234060892 & 2619728737 & 162688610 \\
\hline & $\pi_{S}$ & 298994327 & 6238652375 & 7660505851 & 518008431 & 5918365102 & 354507483 \\
\hline \multirow{4}{*}{ 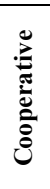 } & $p$ & 1316 & 2832 & 3630 & 1462 & 3189 & 1299 \\
\hline & $A$ & 120106407 & 3219172896 & 4140573039 & 400984045 & 3625150623 & 322261820 \\
\hline & $a$ & 327068546 & 6358042767 & 7683636812 & 439231999 & 5654855668 & 268437867 \\
\hline & $\pi_{S}$ & 447174953 & 9577215662 & 11824209852 & 840216044 & 9280006292 & 590699687 \\
\hline
\end{tabular}

\subsection{Dupolistic retailers models}

For the duopolistic retailers model we use these six groups as shown in Table 5 and obtain the solution as reported in Table 6 . 
Table 5

Six groups of values of parameters considered

\begin{tabular}{lllllll}
\hline & Example 1 & Example 2 & Example 3 & Example 4 & Example 5 & Example 6 \\
\hline$\alpha_{1}$ & 110486 & 124312 & 109599 & 117686 & 112440 & 110696 \\
$\alpha_{2}$ & 111307 & 106890 & 108444 & 119077 & 112440 & 110696 \\
$\beta$ & 41.9926 & 40.8178 & 30.0341 & 40.254 & 35.2557 & 42.6021 \\
$\gamma$ & 11.7373 & 7.3624 & 13.5588 & 8.8159 & 12.5295 & 17.4609 \\
$k_{1}$ & 0.0004977 & 0.0004871 & 0.000443 & 0.0004873 & 0.0004194 & 0.0004557 \\
$k_{2}$ & 0.0003016 & 0.0003466 & 0.0003252 & 0.0004656 & 0.0003358 & 0.0004993 \\
$k_{3}$ & 0.0001253 & 0.0001341 & 0.0001971 & 0.0001598 & 0.0001249 & 0.0001538 \\
\hline
\end{tabular}

Table 6

Three games of values of parameters considered

\begin{tabular}{|c|c|c|c|c|c|c|c|}
\hline & & Example 1 & Example 2 & Example 3 & Example 4 & Example 5 & Example 6 \\
\hline \multirow{10}{*}{$\begin{array}{l}\overline{\bar{n}} \\
\overline{\bar{z}}\end{array}$} & $w$ & 1347 & 1235 & 2597 & 1354 & 1871 & 1700 \\
\hline & $A$ & 274377278 & 305398551 & 1304712274 & 720212541 & 713418073 & 1315788040 \\
\hline & $\pi_{M}$ & 677202181 & 674635938 & 2042041193 & 1134389088 & 1217143566 & 1744297032 \\
\hline & $m_{1}$ & 966 & 1098 & 1432 & 1050 & 1206 & 1003 \\
\hline & $a_{1}$ & 95258260 & 143610915 & 186169606 & 116783675 & 115604449 & 95426910 \\
\hline & $\pi_{R_{1}}$ & 242377252 & 388256194 & 747700778 & 592102451 & 506625855 & 807512274 \\
\hline & $m_{2}$ & 975 & 902 & 1416 & 1065 & 1206 & 1003 \\
\hline & $a_{2}$ & 98685136 & 65471252 & 178145651 & 123869593 & 115604449 & 95426910 \\
\hline & $\pi_{R_{2}}$ & 249297386 & 213314295 & 723911774 & 615754046 & 506625855 & 807512274 \\
\hline & $\pi_{S}$ & 1168876818 & 1276206428 & 3513653745 & 2342245585 & 2230395277 & 3359321581 \\
\hline \multirow{12}{*}{ 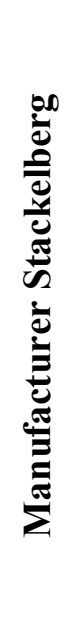 } & $w$ & 1524 & 1477 & 2979 & 1711 & 2214 & 2075 \\
\hline & $A$ & 299587819 & 346783927 & 1406126430 & 834754941 & 788662416 & 1454202890 \\
\hline & $t_{1}$ & 0.4362 & 0.3991 & 0.4405 & 0.4372 & 0.4874 & 0.5219 \\
\hline & $t_{2}$ & 0.4350 & 0.4197 & 0.4375 & 0.4370 & 0.4874 & 0.5219 \\
\hline & $\pi_{M}$ & 742876086 & 724591264 & 2182765553 & 1234834417 & 1337067453 & 1913308359 \\
\hline & $m_{1}$ & 892 & 989 & 1297 & 893 & 1072 & 864 \\
\hline & $a_{1}$ & 217902110 & 261681889 & 399819818 & 193186032 & 274202519 & 229552734 \\
\hline & $\pi_{R_{1}}$ & 234519755 & 357180382 & 646367325 & 466837141 & 438595731 & 641050363 \\
\hline & $m_{2}$ & 901 & 793 & 1281 & 909 & 1072 & 864 \\
\hline & $a_{2}$ & 225386159 & 116125450 & 376819305 & 206893443 & 274202519 & 229552734 \\
\hline & $\pi_{R_{2}}$ & 242255213 & 177398350 & 618780536 & 489758117 & 438595731 & 641050363 \\
\hline & $\pi_{S}$ & 1219651054 & 1259169996 & 3447913413 & 2191429675 & 2214258915 & 3195409084 \\
\hline \multirow{6}{*}{ 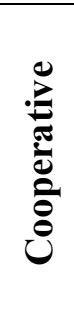 } & $A$ & 939316832 & 1205540338 & 3435784994 & 2692434037 & 2181100000 & 3701338933 \\
\hline & $p_{1}$ & 1832 & 1788 & 3269 & 1878 & 2474 & 2201 \\
\hline & $a_{1}$ & 349353374 & 482642368 & 578377992 & 319411875 & 419390000 & 338299698 \\
\hline & $p_{2}$ & 1833 & 1687 & 3358 & 1887 & 2474 & 2201 \\
\hline & $a_{2}$ & 366795308 & 179369590 & 410981394 & 346929879 & 419390000 & 338300240 \\
\hline & $\pi_{S}$ & 1655465513 & 1867552296 & 4425144380 & 3358775791 & 3019900000 & 4377938871 \\
\hline
\end{tabular}




\section{Managerial implications and Conclusion}

This paper has investigated optimal co-op advertising and pricing decisions in a manufacturer-retailer supply chain with consumer demand, which depends both on the retail price and on the channel members' advertising efforts. We assumed a model recently published by Aust and Buscher (2012) and considered duopolistic retailers in co-op advertising and pricing decisions; we have introduced a new demand function of consumers for each retailer that depends on both co-op advertising and pricing of retailers and manufacturer.

Furthermore, a co-op advertising program is considered, where the manufacturer can accept a certain fraction of the retailer's local advertising costs. By means of game theory, we have analyzed three different relationships within the supply chain: A non-cooperative behavior with equal distribution of power, two situations in which one player dominates his counterpart and cooperation between manufacturer and retailers.

The main contribution of our research is that extends the one manufacturer-one retailer pricing and coop advertising model to the situation with a monopolistic manufacturer and duopolistic retailers. We introduced a promoted demand function of each retailer and investigate the impact of two noncooperative game structures, i.e. Nash game and Manufacturer-Stackelberg game. We develop a cooperative model and show that joint decision can improve the performance of the supply chain. Finally we develop the bargaining game model and shows that how joint extra-profit can be split between players by determine variables $w$ and $t_{i} i=1,2$.

Based on the analysis of the model and results of numerical experiments, we obtain the following insights: 1. Cooperative structure improve the performance of the supply chain and they can gain more profits than non-cooperative situations in both models. 2. In monopolistic model, ManufacturerStackelberg structure gain more profit for both manufacturer and retailer, but in duopolistic retailers model the Nash game and Manufacturer-Stackelberg game solution are close and in some examples Nash game can gain more profit for the supply chain 3 . The highest local advertising expenditure is made in the cooperative and the lowest occurs in the Nash game. When the manufacturer is leader the retailer's spends more on advertising, because the manufacturer participates in local advertising cost $\left(t_{i}>0 \quad i=1,2\right) 4$. We find the coordination mechanism relies on both wholesale price and manufacturer

participation rate $\left(w, t_{i} i=1,2\right)$ where the manufacturer and retailers can bargain to divide the extra-profit accrued from coordination.

There are many research issues that remain to be examined inside the framework of co-op advertising models. First, while our model focused on a single-product chain, the same approach can be used to analyze the multi-product chain by replacement property. Second, we assume a deterministic demand function for each retailer; however with the probabilistic demand in the real word, thus a more interesting issue of future research is suppose a probabilistic demand functions. Third, the forming of coalitions during bargaining seems to be additional motivating field of research.

\section{Acknowledgment}

The authors would like to thank the anonymous referees for their constructive comments on earlier version of this work.

\section{References}

Ahmadi-Javid, A., \& Hoseinpour, P. (2012). On a cooperative advertising model for a supply chain with one manufacturer and one retailer. European Journal of Operational Research, 458-466. 
Aust, G., \& Buscher, U. (2012). Vertical cooperative advertising and pricing decision in a manufacturer-retailer supply chain: A game-theoretic approach. European journal of Operational Research, 473-482.

Bergen, M., \& John, G. (1997). Understanding cooperative advertising participation rates in conventional channels. Journal of Marketing Research 34 (3), 357-369.

Berger, P. (1972). Vertical cooperative advertising ventures. Journal of Marketing research 9 (3), 309312.

Brennan, L. (1988). How retailers are putting it all together? Sales and Marketing Management, 62-65. May.

Cachon, G. (2003). Supply chain coordination with contracts. Handbooks in Operations Research and Management Science, 229-339.

Cachon, G.P., \& Lariviere, M.A. (2005). Supply chain coordination with revenue-sharing contracts: strengths and limitations. . Management Science 51 (1), 30-44.

Choi, S. (1991). Price competition in a channel structure with a common retailer. Marketing Science, 271-296.

He, X., Prasad, A., \& Sethi, S. (2009). Cooperative advertising and pricing in a dynamic stochastic supply chain: Feedback Stackelberg strategies. Production and Operations Management 18 (1), 7894.

Huang Z, \& Li, S. X. (2001). Co-op advertising models in manufacturer-retailer supply chains: A game theory approach. European Journal of Operational Research 135, 527-544.

Huang, Z., \& Li, S. (2005). Coordination and cooperation in manufacturer-retailer supply chains. In: Shi, Y., Xu, W., Chen, Z., et al. Data Mining and Knowledge Management. Springer, Berlin, 174186 (chapter 19).

Huang, Z., Li, S.X., \& Mahajan, V. (2002). An analysis of manufacturer-retailer supply chain coordination in cooperative advertising. Decision Sciences 33 (3), 469-494.

Jorgensen, S., SiguT, S.P., \& Zaccour, G. (2000). Dynamic cooperative advertising in a channel. Journal of Retailing 76 (1), 71-92.

Jorgensen, S., \& Zaccour. (2003). A differential game of retailer promotions. Automatica 39 (7), 11451155.

Jorgensen, S., \& Zaccour, G. (1999). Equilibrium pricing and advertising strategies in a marketing channel. Journal of optimization theory and applications 102 (1), 111-125.

Jorgenson, S. \& Zaccour, G. (2013). Cooperative advertising in marketing channels: game theoretic analysis. Les cahiers du GERAD, 1-22.

Karray, S., \& Zaccour, G. (2006). Could co-op advertising be a manufacturer's counterstrategy to store brands? . Journal of Business Research 59 (9), 1008-1015.

Kebing Chen, T. X. (2011). Ordering policy and coordination of a supply chain with two-period demand uncertainty. European Journal of Operational Research, 347-357.

Li, S.X., Huang, Z., Zhu, J., \& Chau, P.Y.K. (2002). Cooperative advertising, game theory and manufacturer-retailer supply chains. Omega 30 (5), 347-357.

Luo, P. L. (2006). The theory of industrial organization. shanghai: Fundan university Press.

Mingming Leng, A. Z. (2009). Side-payment contracts in two-person nonzero-sum supply chain games:Review, discussion and applications. European Journal of Operational Research, 600-618.

SeyedEsfahani, M.M., Biazaran, M., \& Gharakhani, M. (2011). A game theoretic approach to coordinate pricing and vertical co-op advertising in manufacturer-retailer supply chains. European Journal of Operational Research, 263-273.

Nash, J. (1950). The bargaining problem. Econometrica 18 (2), 155-162.

Roslow, S., Laskey, H.A., \& Nicholls, J.A.F. (1993). The enigma of cooperative advertising. Journal of Business \& Industrial Marketing 8, 70-79.

Wang, S.D., Zhou, Y.W., Min, J., \& Zhong, Y.G. (2011). Coordination of cooperative advertising models in a one-manufacturertwo-retailer supply chain system. Computers \& Industrial Engineering, 1053-1071. 
Szmerekovsky, J., \& Zhang, J. (2009). Pricing and two-tier advertising with one manufacturer and one retailer. European Journal of Operational Research, 904-917.

Xie J., \& Neyret A. (2009). Co-op advertising and pricing models in manufacturer retailer supply chains. Computers \& Industrial Engineering, 1375-1385.

Xie, J. A. (2009). Coordinating advertising and pricing in a manufacturer-retailer channel. European Journal of Operational Research, 758-791.

Yang, S.L., \& Zhou, Y.W. (2006). Two-echelon supply chain models: considering duopolistic retailers' different competitive behaviors. International Journal of Production Economics 103, 104--116.

Yue, J., Austin, J., Wang, M.C., \& Huang, Z. (2006). Coordination of cooperative advertising in a twolevel supply chain when manufacturer offers discount. European Journal of Operational Research $168,65-85$.

\section{Appendix}

$\pi_{M}$ is a strictly Concave function if, for each pair of points on the graph of $\pi_{M}$ the line segment joining these two points lies entirely below the graph of $\pi_{M}$ except at the endpoints of the line segment. In mathematical terminology, $\pi_{M}$ is concave if and only if its $3 \times 3$ Hessian matrix is Negative definite for all possible values of $(A, w, t)$. Hessian matrix is checked for several instances. Since, Hessian matrix is negative define for all instances, so the objective function is concave. The other way to conclude the concavity of the objective function, that we use for ensure the concavity of $\pi_{M}$, is to plot these functions with optional values.

Proof 1: To proof the optimality of these solutions, we calculate the Hessian matrix

$H_{N_{M}}=\left(\begin{array}{ll}\frac{\partial^{2} \pi_{M}}{\partial w^{2}} & \frac{\partial^{2} \pi_{M}}{\partial A \partial w} \\ \frac{\partial^{2} \pi_{M}}{\partial w \partial A} & \frac{\partial^{2} \pi_{M}}{\partial A^{2}}\end{array}\right)$

The second order partial derivatives are as follows:

$$
\frac{\partial^{2} \pi_{M}}{\partial w^{2}}=-2 \beta\left(k_{1} \sqrt{a}+k_{2} \sqrt{A}\right) \quad \frac{\partial^{2} \pi_{M}}{\partial A^{2}}=-\frac{k_{2} w}{4 A^{\frac{3}{2}}}(\alpha-\beta(m+w)) \quad \frac{\partial^{2} \pi_{M}}{\partial A \partial w}=\frac{k_{2}}{2 \sqrt{A}}(\alpha-\beta m-2 \beta w)
$$

The first principle minor of $H_{N_{M}}$ at the solution of Eq. (9) is $H_{N_{M}}^{1}=-2 \beta\left(k_{1} \sqrt{a}+k_{2} \sqrt{A}\right)$ which is always negative. The second principle minor of $H_{N_{M}}$ at the solution of Eq. (9) is

$$
\begin{aligned}
H_{N_{M}}^{2} & =\frac{\partial^{2} \pi_{M}}{\partial w^{2}} \cdot \frac{\partial^{2} \pi_{M}}{\partial A^{2}}-\left(\frac{\partial^{2} \pi_{M}}{\partial A \partial w}\right)^{2}=\left(-2 \beta\left(k_{1} \sqrt{a}+k_{2} \sqrt{A}\right)\right)\left(\frac{-k_{2} w(\alpha-\beta(m+w))}{4 A^{\frac{3}{2}}}\right)-\left(\frac{k_{2}}{2} A^{-\frac{1}{2}}(\alpha-\beta m-2 \beta w)\right)^{2} \\
H_{N_{M}}^{2} & =\frac{\beta^{2}\left(4 \beta k_{1} \sqrt{a}+k_{2}^{2}(\alpha-\beta m)^{2}\right)}{4 k_{2}^{2}\left(\frac{\alpha-\beta m}{2}\right)^{4}}
\end{aligned}
$$

which is always positive. Therefore, the principle minors of $H_{N_{M}}$ have alternating algebraic signs, which means that $H_{N_{M}}$ is negative definite. Hence, $\pi_{M}$ is concave at this specific point, which is therefore a local maximum. As it is the only maximum candidate, we can conclude that it is the 
globally profit maximizing solution of the manufacturer's problem (5). To proof the optimality of Retailers solutions, we have to calculate the Hessian matrix

$$
H_{N_{R}}=\left(\begin{array}{ll}
\frac{\partial^{2} \pi_{R}}{\partial m^{2}} & \frac{\partial^{2} \pi_{R}}{\partial a \partial m} \\
\frac{\partial^{2} \pi_{R}}{\partial m \partial a} & \frac{\partial^{2} \pi_{R}}{\partial a^{2}}
\end{array}\right)
$$

The second order partial derivatives are as follows:

$$
\begin{aligned}
& \frac{\partial^{2} \pi_{R}}{\partial m^{2}}=-2 \beta\left(k_{1} \sqrt{a}+k_{2} \sqrt{A}\right) \\
& \frac{\partial^{2} \pi_{R}}{\partial a^{2}}=\frac{-k_{1} m(\alpha-\beta(m+w))}{4 a^{\frac{3}{2}}} \\
& \frac{\partial^{2} \pi_{R}}{\partial m \partial a}=\frac{k_{2}}{2 \sqrt{a}}(\alpha-2 \beta m-\beta w)
\end{aligned}
$$

The first principle minor of $H_{N_{R}}$ at the solution (9) is $H_{N_{R}}^{1}=-2 \beta\left(k_{1} \sqrt{a}+k_{2} \sqrt{A}\right)$ which is always negative. The second principle minor of $H_{N_{R}}$ is

$$
\begin{aligned}
H_{N_{R}}^{2} & =\frac{\partial^{2} \pi_{R}}{\partial m^{2}} \cdot \frac{\partial^{2} \pi_{R}}{\partial a^{2}}-\left(\frac{\partial^{2} \pi_{R}}{\partial m \partial a}\right)^{2}=\left(-2 \beta\left(k_{1} \sqrt{a}+k_{2} \sqrt{A}\right)\right)\left(\frac{-k_{1} m(\alpha-\beta(m+w))}{\left.4 a^{\frac{3}{2}}\right)}\right)-\left(\frac{k_{2}}{2} a^{-\frac{1}{2}}(\alpha-2 \beta m-\beta w)\right)^{2} \\
H_{N_{R}}^{2} & =\frac{\beta^{2}\left(4 \beta k_{2} \sqrt{A}+k_{1}^{2}(\alpha-\beta m)^{2}\right)}{4 k_{1}^{2}\left(\frac{\alpha-\beta m}{2}\right)^{4}},
\end{aligned}
$$

which is always positive. Therefore, the principle minors of $H_{N_{R}}$ have alternating algebraic signs at the solution (9), which means that $H_{N_{R}}$ is negative definite. Hence, $\pi_{R}$ is concave at this specific point, which is therefore a local maximum. As it is the only maximum candidate, we can conclude that it is the globally profit maximizing solution of the retailer's problem 6 . This completes proof of Theorem 1 .

Proof 2: To proof the optimality of these solutions, we have to calculate the Hessian matrix

$$
H_{S_{M}}=\left(\begin{array}{ccc}
\frac{\partial^{2} \pi_{M}}{\partial w^{2}} & \frac{\partial^{2} \pi_{M}}{\partial A \partial w} & \frac{\partial^{2} \pi_{M}}{\partial t \partial w} \\
\frac{\partial^{2} \pi_{M}}{\partial w \partial A} & \frac{\partial^{2} \pi_{M}}{\partial A^{2}} & \frac{\partial^{2} \pi_{M}}{\partial t \partial A} \\
\frac{\partial^{2} \pi_{M}}{\partial w \partial t} & \frac{\partial^{2} \pi_{M}}{\partial A \partial t} & \frac{\partial^{2} \pi_{M}}{\partial t^{2}}
\end{array}\right)
$$

The second order partial derivatives are as follows:

$$
\frac{\partial^{2} \pi_{M}}{\partial w^{2}}=\frac{3 k_{1}^{2}}{16(1-t)^{2}(\alpha-\beta w)}(\alpha-\beta w)^{2}(-2 \alpha+2 \beta w+2 \alpha t-2 \beta w t-\alpha t+\beta w t) \frac{\beta k_{2} \sqrt{A}}{2(\alpha-\beta w)}(-2 \alpha+2 \beta w)
$$




$$
\begin{aligned}
& \frac{\partial^{2} \pi_{M}}{\partial A^{2}}=-\frac{k_{2} w}{8 A^{\frac{3}{2}}}(\alpha-\beta(m+w)) \quad \frac{\partial^{2} \pi_{M}}{\partial t^{2}}=\frac{k_{1}^{2}}{32 \beta^{2}(1-t)^{4}}(\alpha-\beta w)^{3}(6 \beta w-3 \beta w t-\alpha t-2 \alpha) \\
& \frac{\partial^{2} \pi_{M}}{\partial A \partial w}=\frac{\partial^{2} \pi_{M}}{\partial w \partial A}=\frac{k_{2}}{4 \sqrt{A}}(\alpha-\beta m-\beta w) \quad \frac{\partial^{2} \pi_{M}}{\partial t \partial A}=\frac{\partial^{2} \pi_{M}}{\partial A \partial t}=0 \\
& \frac{\partial^{2} \pi_{M}}{\partial w \partial t}=\frac{k_{1}^{2} w}{16 \beta(1-t)^{3}}(\alpha-\beta w)^{2}(-2 \beta w-3 \beta w+2 \alpha+2 \beta w t+\beta w t)
\end{aligned}
$$

Due to the complexness of the expressions stated above, we are not able to prove the optimality of our solutions analytically. Instead of that, we computed a numerical study with 10000 randomly generated sets of parameters with $70000 \leq \alpha, \alpha_{1}, \alpha_{2} \leq 150000,15 \leq \beta \leq 60,1 \leq \gamma \leq \min \{30, \beta\}, \quad 0.0001 \leq k_{1}, k_{2} \leq 0.0008$, $0.00005 \leq k_{3} \leq \min \left\{0.0003, k_{1}, k_{2}\right\}$ Thereby we could prove numerically, that the principal minors of Hessian matrix $H_{S_{M}}$ always have alternating algebraic signs which means that $H_{S_{M}}$ is negative definite at this specific point. Hence, $\pi_{M}$ is concave in $w, t$ and $A$ at this point, which is therefore a local maximum of the manufacturer's decision problem.

As these solutions are the only roots of the first order partial derivatives Eqs. (11-13) within the considered domain of definition, there is no other extremum candidate and the function cannot change its slope from negative to positive. Therefore, the local optimum stated above also represents the global optimum of $\pi_{M}$.

Proof 3: To proof optimality of our solution, we have to calculate the Hessian matrix

The second order partial derivatives are as follows:

$$
\begin{aligned}
& \frac{\partial^{2} \pi_{S}}{\partial p^{2}}-\beta\left(k_{1} \sqrt{a}+k_{2} \sqrt{A}\right) \quad \frac{\partial^{2} \pi_{S}}{\partial A^{2}}=-\frac{k_{1} p(\alpha-\beta p)}{4 A^{\frac{3}{2}}} \frac{\partial^{2} \pi_{S}}{\partial a^{2}}-\frac{k_{1} p(\alpha-\beta p)}{4 a^{\frac{3}{2}}} \frac{\partial^{2} \pi_{S}}{\partial A \partial p}=\frac{k_{2}(\alpha-2 \beta p)}{2 \sqrt{A}} \quad \frac{\partial^{2} \pi_{S}}{\partial a \partial p}=\frac{k_{1}(\alpha-2 \beta p)}{2 \sqrt{a}} \\
& \frac{\partial^{2} \pi_{S}}{\partial a \partial A}=0
\end{aligned}
$$

The first principle minor of $H_{C}$ at the solution Eq. (17) is:

$$
H_{C}^{1}=\frac{\partial^{2} \pi_{S}}{\partial p^{2}}-\beta\left(k_{1} \sqrt{a}+k_{2} \sqrt{A}\right) \text {, }
$$

which is always negative. The second principle minor of $H_{C}$ at the solution Eq. (17) is:

$$
H_{C}^{2}=\frac{8 \beta^{2}\left(k_{1}^{2}+k_{2}^{2}\right)}{\alpha^{2} k_{1}^{2}}
$$

which is always positive. The third principle minor of $H_{C}$ at the solution Eq. (17) is:

$$
H_{C}^{3}=-\frac{256 \beta^{4}\left(k_{1}^{2}+k_{2}^{2}\right)}{\alpha^{6} k_{1}^{2} k_{2}^{2}}
$$

which is always negative. Therefore, the principle minors of $H_{C}$ have alternating algebraic signs at the solution described by Eq. (17), which means that $H_{C}$ is negative definite. Hence, $\pi_{S}$ is concave at this specific point, which is therefore a local maximum. As it is the only maximum candidate, we can conclude that it is the globally profit maximizing solution. 